\title{
TOWARD THE DEVELOPMENT OF AN INFECTIOUS CDNA CLONE OF A HUMAN ENTERIC CORONAVIRUS
}

\author{
Hongqing Zhu, Yin Liu, Yingyun Cai, Dongdong Yu, Yinghui Pu, \\ Laura Harmon, and Xuming Zhang*
}

\section{INTRODUCTION}

Coronaviruses $(\mathrm{CoV})$ are a large group of plus-strand RNA viruses, which can cause respiratory, digestive, neurological, and immune-mediated diseases in human and animals. The outbreak of the severe acute respiratory syndrome (SARS) in 2003 was caused by SARS-CoV, which is most closely related to group II CoV, especially the bovine CoV $(\mathrm{BCoV})$. Although the exact origin of SARS-CoV remains to be identified, the fact that similar SARS-CoV has been repeatedly isolated in wild animals, including civet cat, raccoon dog, and badges, strongly suggests that SARS-CoV originates from animals. It was postulated that SARS-CoV is a zoonotic pathogen. ${ }^{1}$ Ironically, the zoonotic nature of CoV had been documented long before the outbreak of SARS. In 1994, Zhang et al., ${ }^{2}$ reported the isolation of a human enteric $\mathrm{CoV}(\mathrm{HECoV})$ from a 6-year old child with acute diarrhea. Subsequent characterization of the biologic and antigenic properties and partial sequence of the structural genes revealed that HECoV is more closely related to $\mathrm{BCoV}$ than to other members of the $\mathrm{CoV}$ family. Interestingly, several studies showed that $\mathrm{BCoV}$ was able to infect other small ruminants, turkey, and dogs and cause clinical diseases in these animals, thus further supporting the idea that $\mathrm{BCoV}$ is a zoonotic pathogen. Therefore, $\mathrm{BCoV}$ is the first documented $\mathrm{CoV}$ that can cross species barrier and infect animals ranging from avian, ruminant, carnivore, to humans. In the present study, we attempted to develop an infectious cDNA clone of HECoV by reverse genetics, which will provide a powerful genetic tool for future studies of the molecular evolution and animal to human transmission, and the molecular pathogenesis, and for the design of preventive and therapeutic interventions.

* University of Arkansas for Medical Sciences, Little Rock, Arkansas 72205. 


\section{MATERIALS AND METHODS}

\subsection{Cells and Virus}

The human rectal tumor (HRT)-18 cells and baby hamster kidney (BHK) cells were maintained in Dulbecco's Minimum Essential Medium (DMEM) supplemented with 10\% fetal bovine serum (FBS), which were used for virus propagation and RNA transfection, respectively. The human enteric coronavirus $(\mathrm{HECoV})$ isolate $4408^{2}$ was propagated in HRT-18 cells.

\subsection{Viral RNA Isolation and Reverse Transcription-Polymerase Chain Reaction}

For viral RNA isolation, HRT-18 cells were infected with HECoV at a multiplicity of infection (m.o.i.) of 5 in the presence of actinomycin D. At 48 h p.i., intracellular RNAs were isolated with Trizol reagent according to the manufacturer's protocol (Invitrogen). cDNA was synthesized by reverse transcription (RT) with Maloney murine leukemia virus (M-MLV) reverse transcriptase (Promega) using a primer specific to the nucleotide (nt) sequence at the 3'-end of HECoV nucleocapsid (N) gene ${ }^{2}$ or a nonspecific oligo (dT) primer. Additional 20 pairs of primers were designed for RT and PCR amplification of the entire viral genome. The RT-PCR products were directly sequenced. cDNAs corresponding to the 5'-end of the viral genome was synthesized using the RNAligation-mediated rapid amplification of cDNA end (RLM-RACE) kit (Ambion) and was then sequenced directly. For cloning purpose, PCR was carried out using the Expand Long Taq polymerase (Roche) for 25 cycles. Each cycle consists of $94^{\circ} \mathrm{C}$ for $45 \mathrm{sec}$, $58 \sim 62^{\circ} \mathrm{C}$ for $45 \mathrm{sec}$, and $68^{\circ} \mathrm{C}$ for $3 \sim 4 \mathrm{~min}$ followed by a final extension at $68^{\circ} \mathrm{C}$ for 10 $\min$.

\subsection{Cloning of cDNA Fragments and DNA Sequencing}

For molecular cloning, 9 pairs of primers encompassing the entire viral genome were designed for RT-PCR amplification. The strategy for designing the primers is illustrated in Fig. 1. A T7 promoter was inserted at the most 5'-end primer and a poly (A) tail of 25 As was included at the 3' end primer for the last fragment. An Xho I site was created in the 3' end primer of fragment A without changing the coding amino acid for distinguishing the molecular clone from wild-type virus. A unique BsmB I or Bsa I site was inserted at the terminus of each primer. RT-PCR products were isolated with the gel extract and purification kit (Qiagen) and cloned into Topo II TA vector (Invitrogen) or the pSMART vector (Lucigen).

\subsection{DNA Ligation, in Vitro RNA Transcription, and RNA Transfection}

Each cDNA fragment was released from plasmid DNAs by digestion with appropriate restriction enzymes, separated on $0.8 \%$ agarose gel by electrophoresis, and purified using Qiaex II gel extraction kit (Qiagen). The 9 fragments were then mixed at an equal molar ratio and ligated with T4 DNA ligase (New England Biolab) at $16^{\circ} \mathrm{C}$ overnight. The ligation products were purified by phenol-chloroform-isoamyl and chloroform extraction, and precipitated by ethanol. 
In vitro RNA transcription was carried out using the mMESSAGE, mMACHINE T7 kit (Ambion) with slight modifications. The in vitro transcription products were then treated with RNase-free DNase I (Promega) at $37^{\circ} \mathrm{C}$ overnight, followed by extraction with phenol-chloroform-isoamyl and chloroform, and ethanol precipitation.

For transfection, a mixture of $80 \mu \mathrm{l}$ of the full-length HECoV transcripts and $40 \mu \mathrm{l}$ of the $\mathrm{N}$ gene transcripts was electroporated into BHK cell suspension $\left(10^{6} \mathrm{cell} / \mathrm{ml}\right)$ at $360 \mathrm{~V}$, $80 \mu \mathrm{s}$ in an electroporator (Eppendorf). Transfected BHK cells were seeded onto a dish with HRT-18 cells and the co-culture was incubated at $37^{\circ} \mathrm{C}$ under $5 \% \mathrm{CO} 2$ for 3 days.

\section{RESULTS AND DISCUSSION}

\subsection{Sequencing of the Complete HECoV Genome and Sequence Comparison with Other Related Coronaviruses}

The sequence of the entire $\mathrm{HECoV}$ genome was determined by directly sequencing the RT-PCR products. In some instances, multiple independent RT-PCR products for the same fragments were sequenced. Thus, although quasispecies may exist in the viral populations, the sequence so determined must represent the vast majority of the viral genome in the RNA pools. Analysis of the sequence data revealed that the entire HECoV genome consists of 31,029-nt plus the poly(A) tail. The genome organization is similar to those of other CoV, with the ORFs in the order of 1a-1b-32kDa-HE-S-ORF5-6-7-E-M-N. The 5'-end of the genome is capped as inferred from the result with RLM-RACE and the penultimate $n t$ is guanine. There is a 70-nt leader at the 5'-end, and a core consensus UCUAAAC at the 3 '-end of the leader and the intergenic region proceeding each gene. Sequence comparison with other human $\mathrm{CoV}$ such as $\mathrm{HCoV}-229 \mathrm{E},{ }^{3} \mathrm{HCoV}-\mathrm{OC} 43,{ }^{4}$ SARS-CoV, ${ }^{5}$ and a most recent isolate $\mathrm{HCoV}-\mathrm{NL} 63^{6}$ and animal $\mathrm{CoV}$ revealed that HECoV-4408 is most closely related to $\mathrm{BCoV}^{7}$ with a nt identity of $98 \%$, whereas the nt identity with $\mathrm{HCoV}-\mathrm{OC} 43$ is $95 \%$. Overall, the homology with other three human $\mathrm{CoV}$ was less than $60 \%$. The fact that $\mathrm{HECoV}$ is more closely related the animal $\mathrm{BCoV}$ than to other human $\mathrm{CoV}$ suggests that $\mathrm{HECoV}$ is likely derived from $\mathrm{BCoV}$. This conclusion is consistent with the previous finding obtained from comparative studies on the biologic and antigenic relatedness among $\mathrm{HECoV}, \mathrm{HCoV}-\mathrm{OC} 43$ and $\mathrm{BCoV}{ }^{2}$
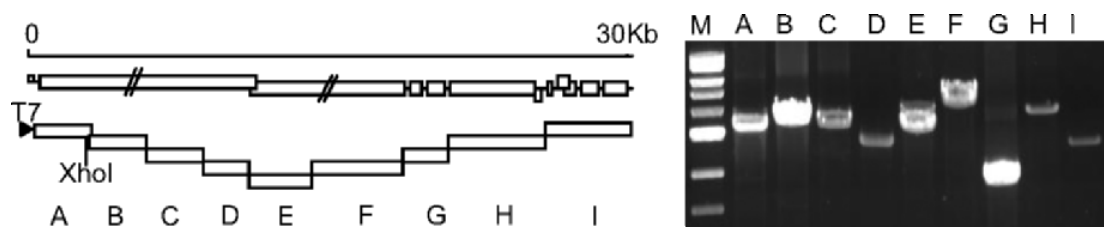

Figure 1. Assembling a full-length cDNA clone of HECoV. cDNA fragments A to I encompass the entire genome $(\approx 32 \mathrm{~kb})$ of $\mathrm{HECoV}$ with indicated open reading frames (left panel) were cloned into plasmids and were released by digestion with BsmB I or Bsa I (right panel). A unique T7 promoter was inserted at the 5' end of fragment A, an Xho I site was created in the fragment A. M, marker for $1 \mathrm{~kb}$ DNA ladder. 


\subsection{Assembly and Confirmation of a Full-Length HECoV cDNA Clone}

To assemble a full-length HECoV cDNA, 9 fragments spanning the HECoV genome was cloned and sequenced. Each cDNA in the plasmids was released by digestion with 2 class II enzymes BsmB I and Bsa I as described, ${ }^{8}$ generating a unique 4-nt overhang at both ends of each fragment. Consequently, only the two neighboring fragments can join together. Thus, a unidirectional assembly of a full-length cDNA was achieved by ligation of the 9 fragments in vitro. RNAs were then transcribed from the cDNAs in vitro with T7 RNA polymerase. Transcription of full-length RNAs was verified by RT-PCR with primers specific to the 3'-end of the viral genome (data not shown). To determine whether the in vitro transcribed, full-length RNA is infectious, we transfected the RNAs together with $\mathrm{N}$ gene transcripts into BHK cells by electroporation and co-cultured the BHK cells with HRT-18 cells for 3 days. Medium from the coculture was blindly passaged for several times in fresh HRT-18 cells. At $72 \mathrm{~h}$ p.i., intracellular RNA was isolated from HRT-18 cells and the synthesis of both genomic and subgenomic mRNAs were determined by RT-PCR using primers specific to genomic fragment A or to the 5'end of $\mathrm{N}$ mRNA. Indeed, both fragments were amplified. Fragment A was digested with XhoI. Because an Xho I site was engineered specifically into fragment A, this indicates that the RNAs present in HRT-18 cells are derived from transfected RNA. In contrast, although a similar fragment A was amplified from HECoV-infected HRT-18 cells (which used as a positive control), it could not be digested with XhoI (data not shown). These data demonstrate that the full-length HECoV cDNA clone is infectious in HRT-18 cells. The generation of a full-length $\mathrm{HECoV}$ infectious cDNA clone will provide a powerful approach for genetic manipulation of the $\mathrm{HECoV}$ genome, for studying viral pathogenesis, and for developing potential vaccines.

\section{ACKNOWLEDGMENTS}

This work was supported by grants from the National Institutes of Health (AI 59244 and AI61204).

\section{REFERENCES}

1. Guan, Y., Zheng, B.J., He, Y.Q., et al., 2003, Isolation and characterization of viruses related to the SARS coronavirus from animals in southern China, Science 302:276-278.

2. Zhang, X. M., Herbst, W., Kousoulas, K. G., and Storz, J., 1994, Biological and genetic characterization of a hemagglutinating coronavirus isolated from a diarrhoeic child, J. Med. Virol. 44:152-161.

3. Thiel, V., Herold, J., Schelle, B., and Siddell, S. G., 2001, Infectious RNA transcribed in vitro from a cDNA copy of the human coronavirus genome cloned in vaccinia virus, J. Gen. Virol. 82:1273-1281.

4. Vijgen, L., Keyaerts, E., Moes, E., et al., 2005, Complete genomic sequence of human coronavirus OC43: molecular clock analysis suggests a relatively recent zoonotic coronavirus, J. Virol. 79:1595-1604.

5. Rota, P.A., Oberste, M.S., Monroe, S.S., et al., 2003, Characterization of a novel coronavirus associated with severe acute respiratory syndrome, Science 300:1394-1399.

6. Van Der Hoek, L., et al., 2004, Identification of a new human coronavirus, Nat. Med. 10:368-373.

7. Chouljenko, V.N., Lin, X.Q., Storz, J., Kousoulas, K.G., and Gorbalenya, A.E., 2001, Comparison of genomic and predicted amino acid sequences of respiratory and enteric bovine coronaviruses isolated from the same animal with fatal shipping pneumonia, J. Gen. Virol. 82:2927-2933.

8. Boyd, Y., Denison, M., Weiss, R.W., and Baric, R.S., 2002, Systematic assembly of a full-length infectious cDNA of mouse hepatitis virus strain A59, J. Virol. 76:11065-11078. 